\title{
Effects of regular exercise plus food restriction on left ventricular pathological remodeling in heart failure-induced rats
}

\author{
Moradi $\mathrm{F}^{1}$, Imani $\mathrm{AR}^{2}$, Faghihi $\mathrm{M}^{2}$ \\ Department of Physiology, School of Medicine, Tehran University of Medical Sciences, Tehran, \\ Islamic Republic of Iran. faghihim@tums.ac.ir
}

\begin{abstract}
BACKGROUND: Cardiac remodeling is the main pathophysiological process leading to heart failure. Exercise and food restriction have been shown to exert some profound physiological benefits.

OBJECTIVES: This study investigated the effects of exercise plus food restriction (FR) on rat left ventricular remodeling.

METHODS: Fifty male rats were randomly divided into 5 groups. 1) Sham (saline injection), 2) ISO (isoproterenol injection), 3) FR+ ISO (8 weeks with $60 \%$ food restriction and then isoproterenol injection), 4) E+ISO (run-in period of 4 weeks on treadmill and then isoproterenol injection), and 5) $F R+E+I S O$. Serum levels of creatine kinase, nitric oxide, gene expression of microtubule-associated protein 1 light chain 3-I and II, Beclin-1, Bax and $\mathrm{Bcl} 2$ and TUNEL staining were investigated.

RESULTS: ISO increased the plasma CK-MB level, gene expression of Bax and TUNEL-positive cells in left ventricle and at the same time, decreased the serum level of NO. Regular exercise plus food restriction enhanced the expression of LC3B-II, Beclin-1, Bcl2 genes and elevated LC3B-II / LC3B-I, while decreasing the gene expression of Bax and TUNEL-positive cells in the left ventricle.

CONCLUSION: Our results propose that exercise plus food restriction is more effective than either therapy alone for possibly preserving cardiac internal defenses against heart failure consequences and remodeling (Tab. 2 , Fig. 3, Ref. 20). Text in PDF www.elis.sk.

KEY WORDS: heart failure, food restriction, exercise, apoptosis, autophagy.
\end{abstract}

\section{Introduction}

Cardiac remodeling is the main pathophysiological process leading to heart failure (HF) and characterized with maladaptation to myocardial stress at molecular, cellular, tissue, and organ levels. The rate of incidence of much cardiovascular pathology is directly related to a number of interconnected factors such as life habits, stress, aging and other public health-related criteria in many societies $(1,2)$. Recent experimental studies have shown that food restriction can prolong lifetime and decrease age-related cardiovascular pathologies (3). Both food restriction and physical exercise are also known to improve the cardiac function in obese individuals (4). However, the precise impact of food restriction and simultaneous chronic physical training on cardiac contractile function and cellular viability has not been well evaluated.

In fact, the rising tide of obesity by unhealthy diets in industrialized societies and development of sedentary professions have

${ }^{1}$ Physiology Department, Faculty of Medicin, Zanjan University of Medical Sciences, Zanjan, Iran, and ${ }^{2}$ Physiology Department, Faculty of Medicin, Tehran University of Medical Sciences, Tehran, Iran

Address for correspondence: M. Faghihi, Department of Physiology, School of Medicine, Tehran University of Medical Sciences, Tehran, Islamic Republic of Iran.

Phone: +98.21.66419484, Fax: +98.21.66419484

Acknowledgement: This study was financially supported by Tehran University of Medical Sciences project number: 25796 (Tehran, Iran). resulted in an increase in the prevalence of cardiovascular events $(5,6)$. It seems that two interventions that reliably reduce the blood pressure and improve glucose metabolism in humans are diet restriction (7) and physical exercise (8). Exercise has been well established to improve the peripheral alterations associated with HF, but little research has explored the use of exercise with or without food restriction as an adjunct therapy for HF.

Autophagy is essential to cell survival. Its interruption triggers ventricular dysfunction and heart failure (9). Although several molecular mechanisms of ventricular remodeling have been elucidated, there is much that still remains unknown. Adverse remodeling of the ventricle involves changes in the balance between cellular protein synthesis and degradation, hence, forcing the myocytes in a delicate equilibrium between life and death. In this context, the process of macroautophagy has been recognized to play a direct role in the pathophysiology of heart failure $(10,11)$.

Lifestyle interventions such as caloric restriction and exercise have been shown to improve obesity and the associated risk of diseases. Now an important equation remains to be clarified, namely as to which of them are the safest and most effective to provide and strengthen body defenses in pathological situations and maintain better response in at-risk individuals, e.g. attenuation of heart failure consequences. To better evaluate the effect of food restriction and exercise training on heart function under physiological conditions, a noninvasive myocardial infarction rat model was used to assess the efficacy of food restriction with or 
Tab. 1. Experimental grouping: groups, ISO-induced heart failure and the animal tasks which were conducted.

\begin{tabular}{lcccc}
\hline Groups & $\begin{array}{c}\text { Exercise on } \\
\text { treadmill } \\
(4 \text { weeks })\end{array}$ & $\begin{array}{c}\text { Food } \\
\text { restriction } \\
(60 \%)\end{array}$ & $\begin{array}{c}\text { Saline } \\
\text { injections }\end{array}$ & $\begin{array}{c}\text { Isoproterenol } \\
\text { injections }\end{array}$ \\
\hline sham & - & - & + & - \\
ISO & - & - & - & + \\
FR+ISO & - & + & - & + \\
E+ISO & + & - & - & + \\
FR+E+ISO & + & + & - & + \\
\hline
\end{tabular}

without exercise on protecting the heart against cell death overactivation and focus on the correlated gene expression of autophagic factors and histopathological changes.

\section{Materials and methods}

\section{Experimental animals}

The experimental protocol was approved by the institutional care and use committee of Tehran University of Medical Sciences (Tehran, Iran) and EU (86/609/EEC). In this experiment, 50 male Wistar rats (weighing 250-300 g) were housed under controlled environment conditions $\left(22 \pm 2{ }^{\circ} \mathrm{C}\right.$; light cycle $\left.7 \mathrm{AM}-7 \mathrm{PM}\right)$. Rats were allowed to access water and standard laboratory food ad libitum. Animals were adapted for at least 6 days before the experiments.

\section{Animal grouping}

The animals were divided in five experimental groups as shown in Table $1(\mathrm{n}=10)$.

\section{Heart failure induction}

To induce experimental heart failure, after 8 weeks of study, isoproterenol was dissolved in normal saline and injected subcutaneously to rats $(130 \mathrm{mg} / \mathrm{kg})$ daily for 2 consecutive days (12).

\section{Exercise training protocol}

The rats in the training intervention groups were trained to run on a treadmill as described before (13; 4-lane animal treadmill; IITC Life Science Inc., USA). They had one session daily, 5 days a week for 4 weeks.

\section{Biochemical analysis}

Blood samples were collected from retro-orbital plexus on days 1,3 and 5 after isoproterenol injections for biochemical analysis (NO and $\mathrm{CK}-\mathrm{MB}$ ). The samples were centrifuged at 5,000 rpm, and $4{ }^{\circ} \mathrm{C}$, for $15 \mathrm{~min}$. Serum levels of CK-MB (marker of myocyte necrosis) were measured by a colorimetric method, and serum $\mathrm{NO}$ metabolites, nitrite $\left(\mathrm{NO}^{2-}\right)$ and nitrate $\left(\mathrm{NO}^{3-}\right)$ were measured as an index of NO production, based on the Griess reaction.

\section{RNA isolation and semi -quantitative real-time PCR assay}

Finally, after anesthetizing the animals with chloroform, the chest was opened and the heart was taken out and put on an icecooled cutting board. Left ventricles were dissected, snapped frozen in liquid nitrogen and stored at $-70^{\circ} \mathrm{C}$ for extraction of RNA. According to the manufacturer's protocol, total RNA in LV samples was isolated with Trizol reagent (Invitrogen, Shanghai, China). For cDNA synthesis, $1.0 \mathrm{mg}$ RNA was used, and reactions were carried out using reverse transcribed system (Takara, Japan). RT-PCR was performed in a GeneMate thermal cycler (Jinge Instr, Hangzhou, China). Quantification of gene expression was performed using the Rotor-Gene 6000 (Qiagene). The value for each sample was an average of three independent PCR measurements. Quantitative real-time PCR was performed using SYBR Green Master Mix (Takara Bio, Inc.) The cycle threshold (CT) values were automatically determined in triplicate and averaged. All real-time PCR sample reactions were normalized to HPRT as a housekeeping expression. A standard curve was run with the dilution series of the amplified fragment allowing for mRNA copy number calculation. The relative expression of Bax and Bcl2, LC3-I, LC3-П and Beclin-1 as were calculated using the ${ }^{-}{ }^{\Delta \Delta C T}$ method (14). The specific primer sequences are listed in Table 2.

Terminal deoxynucleotidyl transferase-mediated dUTP nick endlabeling (TUNEL) staining and counting

Once the physiological measurements were complete on 30th day of isoproterenol injections, the rats were subjected to deep anesthesia and sacrificed. The hearts were removed and subsequently put in tissue processor (DS 2080/H, Tehran, Iran) for processing. Afterwards, they were embedded in paraffin for histopathological evaluation. TUNEL was performed on 4-5 $\mu \mathrm{m}$-thick paraffinembedded sections using the In Situ Cell Death Detection Kit, POD (Roche Applied Science, Germany). In this procedure, nuclei undergoing apoptosis were labeled with green fluorescence. The number of TUNEL-positive cells was manually counted in five randomly selected fields of view under $400 \times$ magnification for each animal and five animals were studied per group.

\section{Statistical analysis}

Data were analyzed using both one-way ANOVA and two-way ANOVA, followed by Tukeys' post hoc statistical test for normal data or Kruskall-Wallis tests followed by Mann-Whitney's U test for non-parametric data. All data were expressed as mean \pm SEM. The value of $\mathrm{p}<0.05$ was accepted as statistically significant. The changes in fold of gene expressions were calculated with Rest software, version 2009.

\section{Tab. 2. The sequence of primers used in Real-Time PCR.}

\begin{tabular}{llc}
\hline $\begin{array}{l}\text { Gene } \\
\text { name }\end{array}$ & Primer sequence PCR & $\begin{array}{c}\text { product } \\
\text { size }\end{array}$ \\
\hline LC3-I & $\begin{array}{l}\text { Forward primer: TGCAGCAGATCCGTGACCA } \\
\text { Reverse primer: GTGGGGTTGAGTTGCAGGC }\end{array}$ & 148 \\
\hline LC3-П & $\begin{array}{l}\text { Forward primer: CGGGTTGAGGAGACACAC } \\
\text { Reverse primer: AGCCGGACATCTTCCACTCT }\end{array}$ & 181 \\
\hline Beclin-1 & $\begin{array}{l}\text { Forward primer: CATTCAGGAACTCACAGCTCCA } \\
\text { Reverse primer: ACACCATCCTGGCGAGTTTC }\end{array}$ & 457 \\
\hline Bax & $\begin{array}{l}\text { Forward: GGCGAATTGGCGATGAACTG } \\
\text { Reverse: ATGGTTCTGATCAGCTCGGG }\end{array}$ & 217 \\
\hline Bc12 & $\begin{array}{l}\text { Forward: CATCGCTCTGTGGATGACTGA } \\
\text { Reverse: CTGGGGCCATATAGTTCCACAA }\end{array}$ & 99 \\
\hline HPRT & $\begin{array}{l}\text { Forward primer: CTCATGGACTGATTATGGACAGGAC } \\
\text { Reverse primer: GCAGGTCAGCAAAGAACTTATAGCC }\end{array}$ & 123 \\
\hline
\end{tabular}



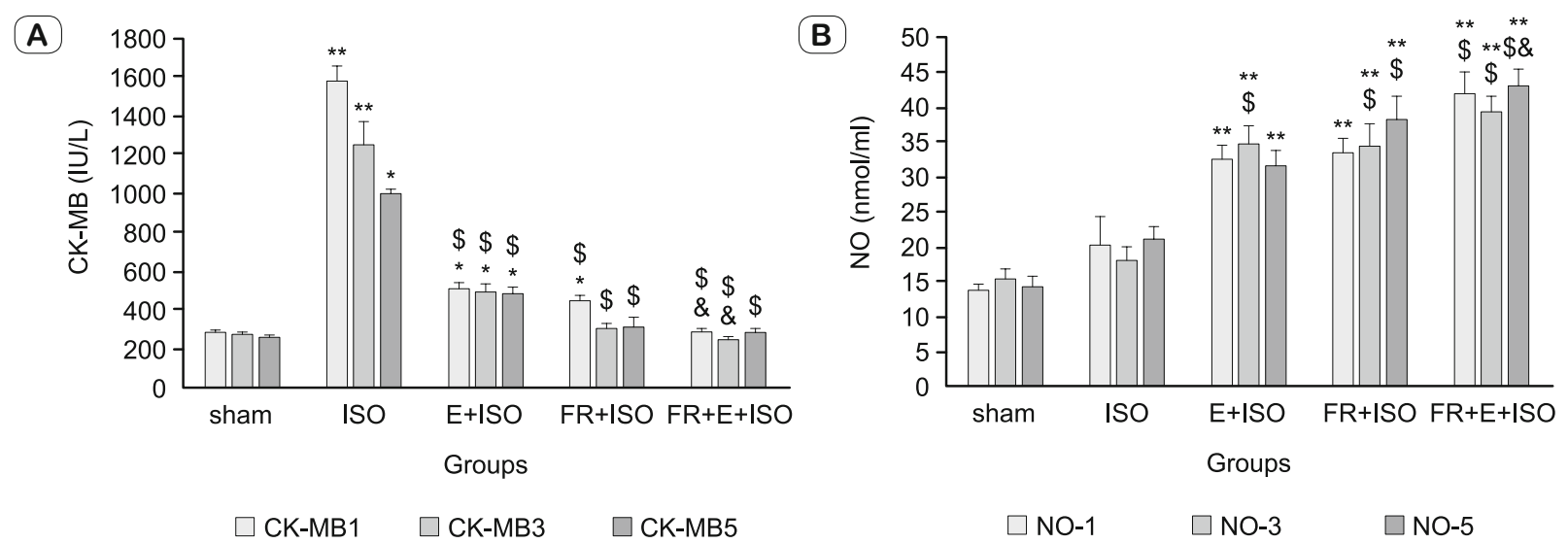

Fig. 1. A) Analysis of serum levels of CK-MB on days 1, 3 and 5 after injections of isoproterenol and B, Serum levels of NO on days 1, 3 and 5 after injections of isoproterenol using two-way ANOVA $(\mathrm{n}=10)$. Data are presented as means \pm SEM. *p $<0.05 \mathrm{vs} \mathrm{sham,} * * \mathrm{p}<0.001 \mathrm{vs}$ sham, ${ }^{s}$ p $<0.05$ vs ISO, ${ }^{\&}$ p < 0.05 vs FR+ISO and vs E+ISO. ISO: isopretrenol; FR: food restriction; E: exercise.

\section{Results}

\section{CK-MB levels}

Induction of HF significantly increased serum levels of CKMB in ISO group in comparison with sham group on days $1(\mathrm{p}<$ $0.001), 3$ ( $\mathrm{p}<0.001)$ and 5 ( $\mathrm{p}<0.05$ ) after injections (Fig. 1A). Our results not only showed that food restriction and exercise before induction of $\mathrm{HF}$ gradually decreased CK-MB levels to those in sham group in the consecutive days but also showed that latter levels in FR+E+ISO rats were lower than those in FR+ISO and E+ISO groups. As shown in Figure 1A, on various days until 5th day, the CK-MB levels remained relatively constant.

\section{NO levels}

Pretreatment with food restriction and regular exercise before induction of HF significantly increased serum levels of NO in comparison with sham group on days 1,3 and $5(\mathrm{p}<0.001)$ and ISO group $(\mathrm{p}<0.05)$ (Fig. 1B). Our results also showed that food restriction in conjunction with exercise significantly increased NO levels in comparison with $\mathrm{E}+\mathrm{ISO}$ group on the 5th day after injections.

\section{mRNA levels of autophagic and apoptotic genes}

Pretreatment with regular exercise significantly increased expression of LC3-I, LC3-П and Beclin-1 ( $p<0.05)$ when compared to sham and ISO animals. The expression of all these autophagic factors in FR+E+ISO animals was significantly higher when compared with sham, ISO, E+ISO and FR+ISO groups $(\mathrm{p}<$ 0.05 ). Moreover, in comparison with sham and ISO animals, the gene expressions of $\mathrm{Bcl} 2$ as an antiapoptotic agent was increased in all food-restricted animals $(\mathrm{p}<0.05)$. The $\mathrm{Bcl} 2$ gene, especially in $\mathrm{FR}+\mathrm{E}+\mathrm{ISO}$ rats, showed more overexpression $(\mathrm{p}<0.001)$. In contrast, the expression of Bax gene as a proapoptotic factor was highest in ISO rats ( $p<0.001$ when compared to sham group). Our data analysis showed no significant differences in mRNA expression between ISO and sham animals.

\section{Apoptosis in myocytes}

Representative photomicrographs of the TUNEL-stained tissue sections of experimental groups are shown in Figure 3. No evidence of TUNEL reaction was detected in the sham animals. The administration of isoproterenol in ISO group caused a significant accumulation of TUNEL-positive cells in ventricular sections in comparison with the sham group $(\mathrm{p}<0.001)$. Also, the preconditioning with exercise training for 4 weeks and $60 \%$ food restriction attenuated the accumulation of apoptotic bodies in the heart tissues ( $<$ 0.05) (Fig. 4).

\section{Discussion}

The main findings of this study are that in experimental model of isoproterenol-induced heart failure, the left ventricle showed a significant increase in plasma CK-MB level and gene expression of Bax and TUNEL-positive cells as apoptotic factor. Also, the defensive impact of food restriction and exercise training on myocardial damage was verified by means of increased serum level of NO and decreased CK-MB. A considerably significant increase in expression of LC3-II (and LC3-II to LC3-I ratio) and Beclin-1 took place. Therefore, cardiac autophagy took place following food restriction and exercise training. Caloric restriction has been described as a subtraction in calories received below the average ad libitum intake while still causing no malnutrition (15). In the following study, the amount of food received was reduced to $60 \%$ of the average food intake of sham animals. To respond to the apparent lack of energy, rats showed an obvious reduction in their body weight, fat mass and heart weight (data not shown). Studies indicated that the activation of autophagy is an important key finding stemming from the pathophysiology of heart failure. At lower levels, autophagy performs housekeeping functions, maintaining cardiomyocyte function and ventricular mass. A couple of questions come into our minds, namely as to whether a more intensive autophagic response leads to cardiomyocytes' failure of repairment and as to whether autophagy can be considered a protective mechanism or a process contributing 
243-248

A

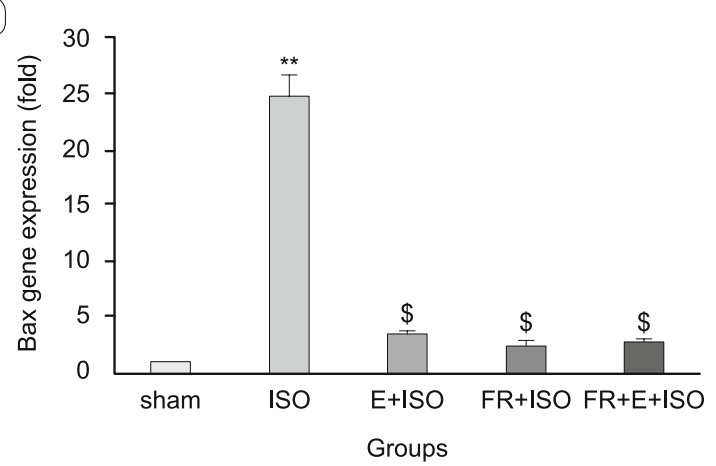

C

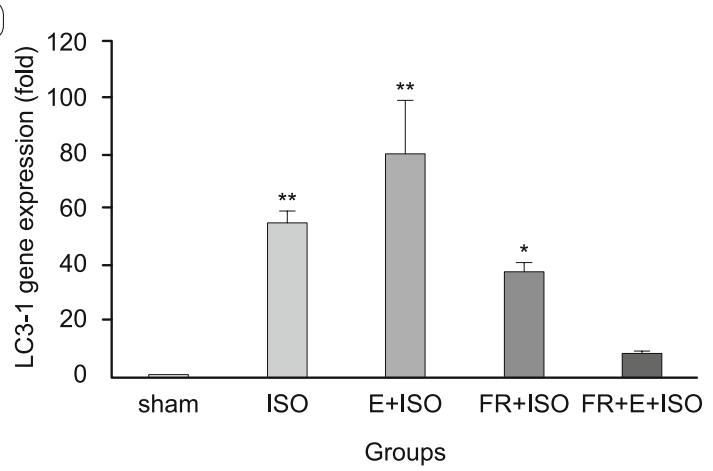

(E)

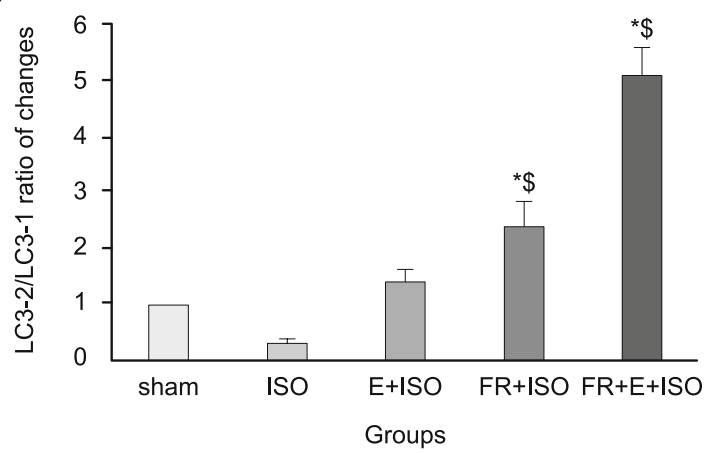

B

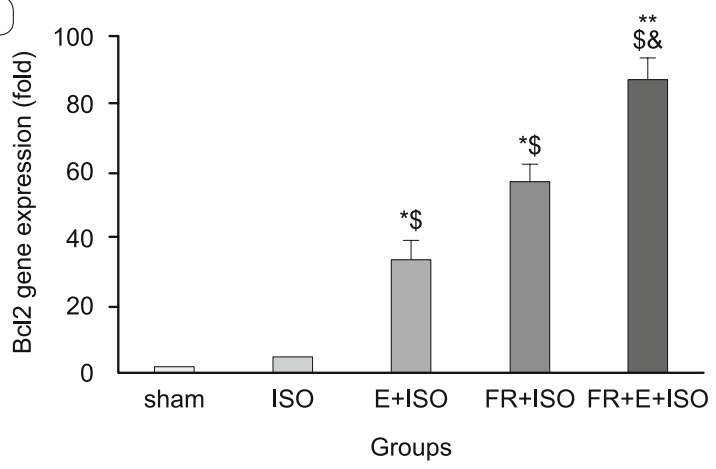

(D)

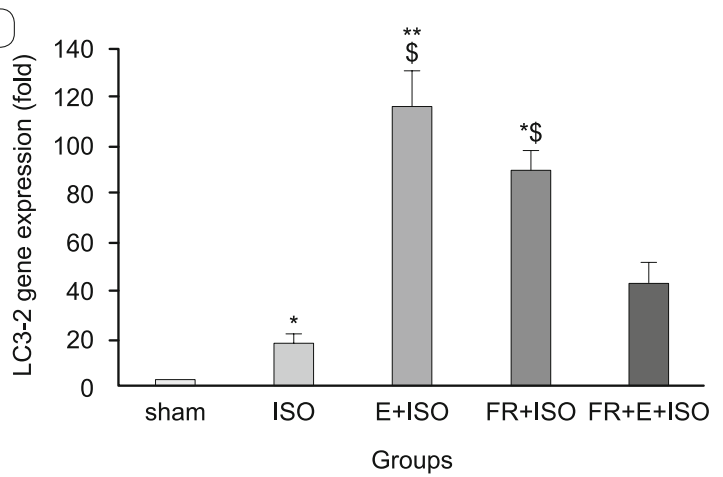

F

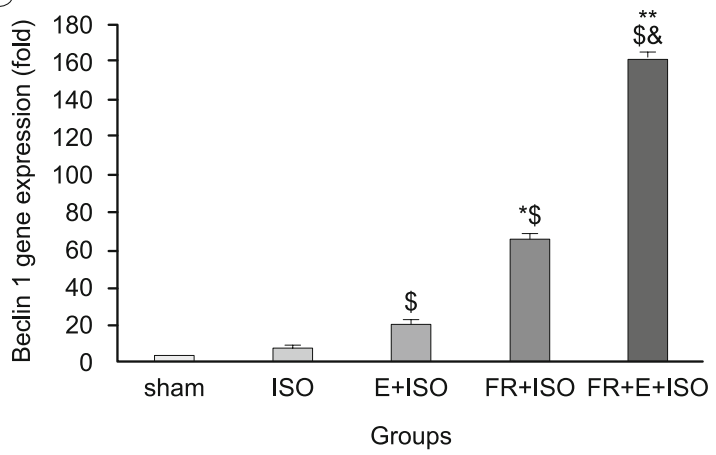

Fig. 2. Analysis of A: Bax; B: Bcl2; C: LC3-I; D: LC3-П; E: LC3-П/LC3-I ratio and F: Beclin-1 mRNA expression in the heart using one-way ANOVA (n=5). Data are presented as means \pm SEM. ${ }^{*} p<0.05$ vs sham, ${ }^{* *} p<0.001$ vs sham, ${ }^{\$} p<0.05$ vs ISO, ${ }^{\&} p<0.05$ vs FR + ISO and vs E+ISO. ISO: isopretrenol; FR: food restriction; E: exercise.

to disease progression. Autophagy also happens when the human heart fails while upregulations have been reported in animal models of pressure overload-induced heart failure. Although the factors that determine whether autophagy will play a protective or harmful role are not well known, the level and duration of autophagy seem important. Autophagy might antagonize ventricular hypertrophy by enhancing protein degradation, thus decreasing the tissue mass. However, the rate of protective effects of autophagy attenuates with some factors. Accordingly, the development of benefit advices in life style changes such as food restriction or physical activity via promoting the qualities and quantities of the autophagic process and attenuating the cell death aspects would be of precise value in the treatment of heart failure (16). In addition, current evidence indicates that autophagic activity under physiological conditions is important for cellular homeostasis, whereas excessive autophagy is rather destructive (17). As already mentioned above, autophagy functions predominantly conceived as a pro-survival pathway during nutrient deprivation and other forms of cellular stress. However, when autophagy is severely triggered, the autophagic machinery may also be used for self-destruction and death. Hence, when autophagic cell death is triggered in cardiac cells, it may contribute to the worsening of heart failure (16). Caloric restriction has been demonstrated to improve the performance of many organs such as that of cardiac function in humans and experimental animal models (17). Our study reveals that long-term caloric restriction preserved myocardial contractile function, improved cardiomyocyte function and induced cardiomyocyte autophagy, while lessening the remodeling process. In the current study of the levels of autophagy-related 
TUNEL
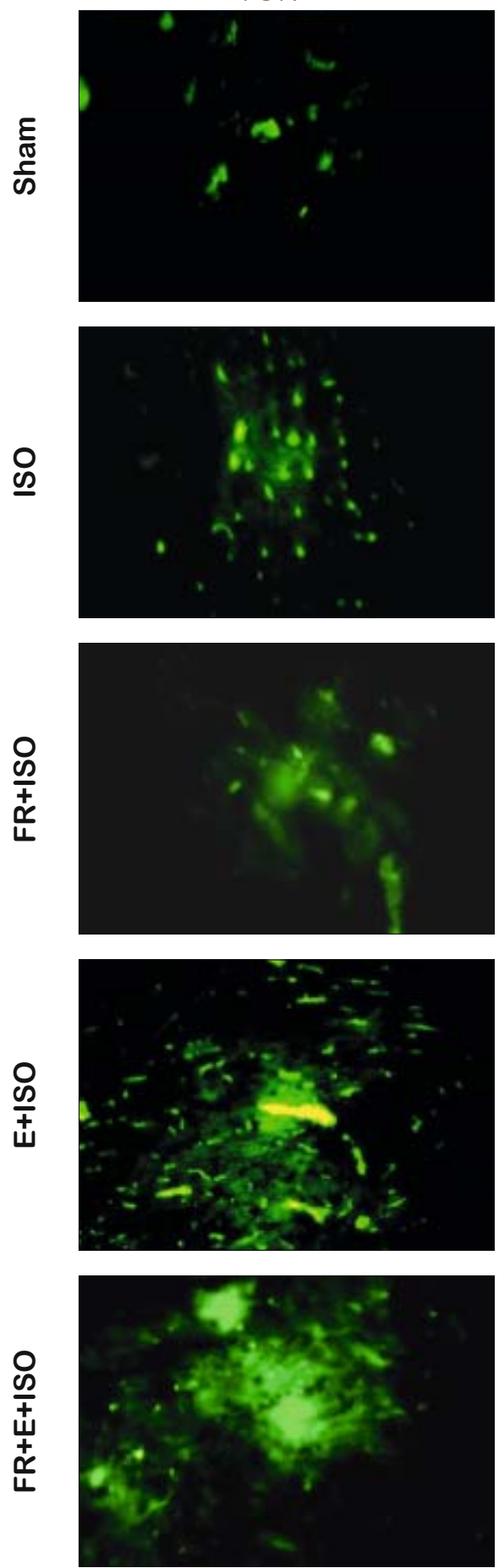

DAPI
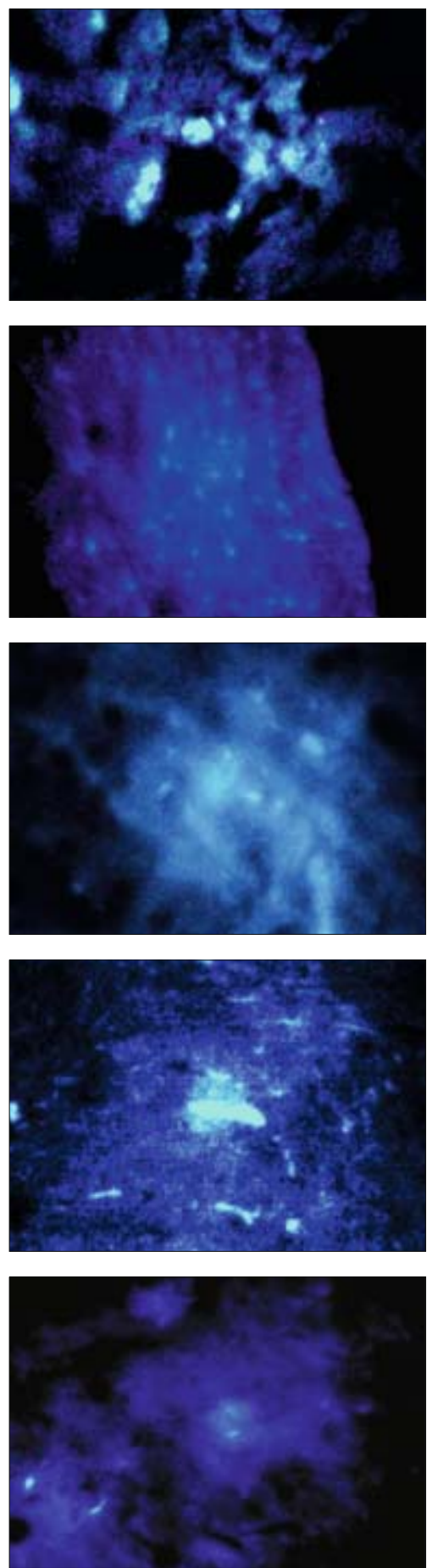
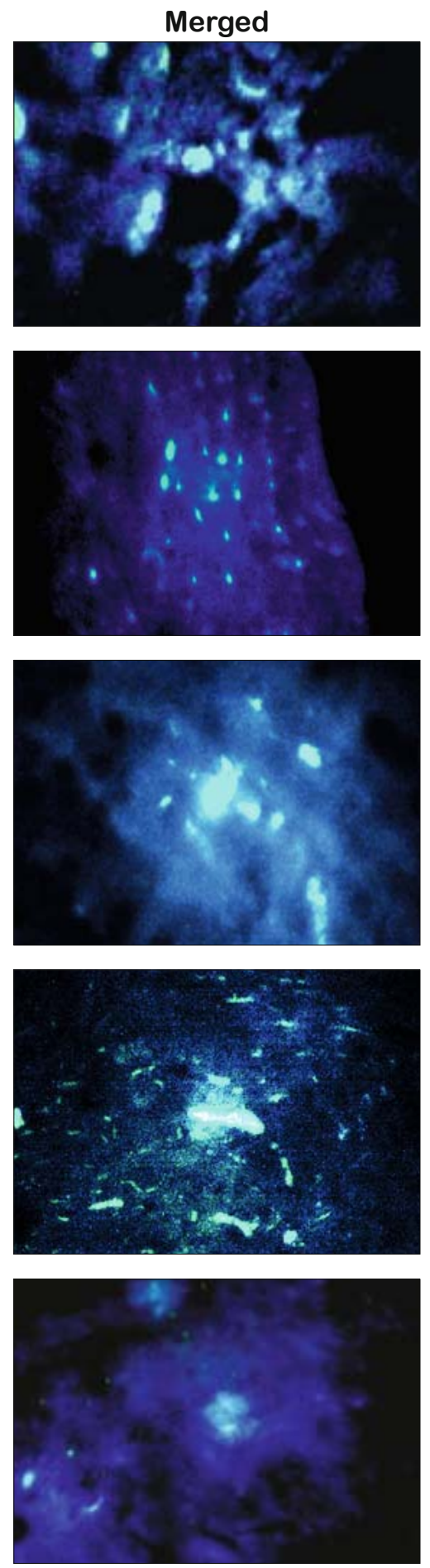

Fig. 3. A: Representative photomicrographs of TUNEL staining of ventricular sections. Apoptotic cells (green) and DAPI staining of the DNA (blue) by fluorescence microscope, (Scale bar: $25 \mu \mathrm{m}$ )

proteins, the gene expression was found to be elevated (LC3-II and Beclin-1) in hearts following 4 weeks of exercise training and caloric restriction. Our findings support the notion that constitutive cardiomyocyte autophagy is needed for protein quality control, normal cellular structure and function in heart failure situation.
Similar to our results, Wohlgemuth and colleagues reported that lifelong $40 \%$ caloric restriction drastically increased the expression of autophagic markers in the heart (18). The amount of conjugated LC3 (LC3-II) correlates with the number of autophagosomes. Contemporary studies report that an increase in the ratio of LC3-II to 


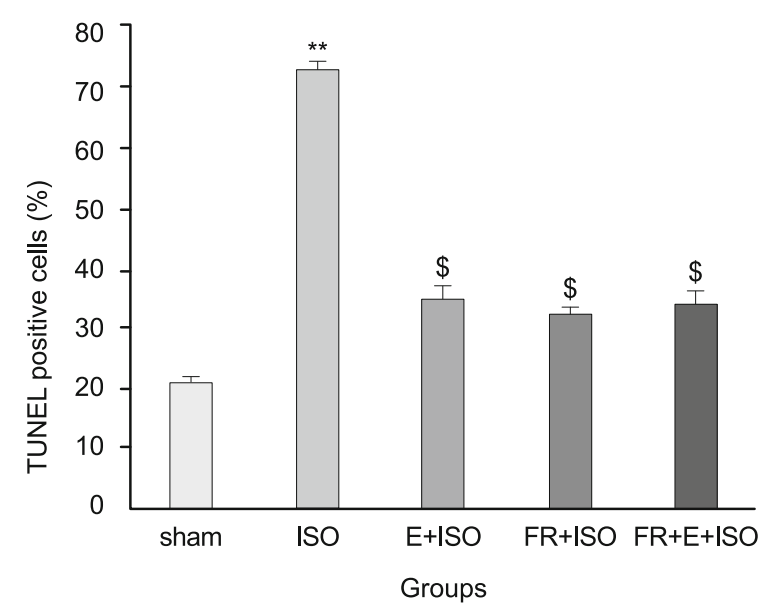

Fig. 3. B: Quantification of TUNEL positive cells using one-way ANOVA $(n=5)$. Data are presented as means \pm SEM. **p $<0.05$ vs sham, ${ }^{\$} p<0.05$ vs ISO. ISO: isopretrenol; FR: food restriction, E: exercise.

the cytosolic form of LC3 (LC3-I) is a better biochemical marker by which to assess the ongoing autophagy (19). Although the expression levels of LC3-I were similar among ad libitum sham rats and FR, the expression of LC3-II was significantly higher in hearts of FR and E rats. Consequently, an increasing ratio of LC3-II to LC3-I was seen in the hearts of food-restricted and exercising rats (Fig. 2E). In addition, the expression levels of Beclin 1 were higher in hearts from FR rats compared with those in the hearts from sham rats (Fig. 2F). However, the LC3-II/LC3-I ratio remained higher in the hearts from FR and $\mathrm{E}$ rats compared with sham rats, thus suggesting that the autophagic flux is enhanced in the hearts of FR and $\mathrm{E}$ rats. Mitochondria in aged post-mitotic cells are enlarged and structurally deteriorated, showing a swelling, loss of cristae, and deficient ATP production (9). These senescent-like abnormalities of mitochondria in autophagy-deficient cardiomyocytes might be responsible for cardiac dysfunction in ISO rats. Mitochondrial activity is a major source of endogenous reactive oxygen species (ROS) causing oxidative damage of cytosolic materials. We detected increases in the number of apoptotic cardiomyocytes in ISO rat hearts (Fig. 3). An increase in oxidative stress in the heart can lead to cardiomyopathy (20). It is possible that the accumulation of abnormal mitochondria results in increased oxidative stress and leads to cardiomyocyte apoptosis. Recent study showed that in the left ventricular myocardium of patients with idiopathic dilated cardiomyopathy, mechanical unloading significantly decreases mRNA transcript levels of Beclin-1, Atg5, and LC3. Protein levels of Beclin-1, Atg5-Atg12 conjugate, and LC3-II are also reduced after left ventricular assist device support. Thus, mechanical unloading of the failing heart decreases markers of autophagy. This further indicates that autophagy may be a maladaptive mechanism in the failing heart (9).

This study suggests that the preconditioning with $60 \%$ food restriction and regular physical training lessens the cardiac remodeling under physiological state. This possibly takes place through modulation of the autophagic state, precisely through Beclin1, LC3 and apoptosis signaling cascades. These results suggest that continuous constitutive autophagy plays a crucial role in maintaining cardiac structure and function.

\section{References}

1. Alla F, Zannad F, Filippatos G. Epidemiology of acute heart failure syndromes. Heart Failure Rev 2007; 12 (2): 91-95.

2. Aronow WS. Epidemiology, pathophysiology, prognosis, and treatment of systolic and diastolic heart failure. Cardiol Rev 2006; 14 (3): 108-124.

3. Han X, Ren J. Caloric restriction and heart function: is there a sensible link? Acta Pharm Sinica 2010; 31 (9): 1111-1117.

4. Mercken EM, Carboneau BA, Krzysik-Walker SM, de Cabo R. Of mice and men: the benefits of caloric restriction, exercise, and mimetics. Ageing Res Rev 2012; 11 (3): 390-398.

5. Wan R, Camandola S, Mattson MP. Intermittent fasting and dietary supplementation with 2-deoxy-D-glucose improve functional and metabolic cardiovascular risk factors in rats. FASEB J 2003; 17 (9): 1133-1134.

6. Sharma AM. The value of current interventions for obesity. Nat Clin Pract Cardiovasc Med 2008; 5: S3-S9.

7. O'Keefe JH, Gheewala NM, O'Keefe JO. Dietary strategies for improving post-prandial glucose, lipids, inflammation, and cardiovascular health. J Amer Coll Cardiol 2008; 51 (3): 249-255.

8. Lippincott MF, Carlow A, Desai A, Blum A, Rodrigo M, Patibandla S et al. Relation of endothelial function to cardiovascular risk in women with sedentary occupations and without known cardiovascular disease. Amer J Cardiol 2008; 102 (3): 348-352.

9. Nishida K, Yamaguchi O, Otsu K. Crosstalk between autophagy and apoptosis in heart disease. Circ Res 2008; 103 (4): 343-351.

10. Levine B, Yuan J. Autophagy in cell death: an innocent convict? J Clin Invest 2005; 115 (10): 2679-2688.

11. Thorburn A. Apoptosis and autophagy: regulatory connections between two supposedly different processes. Apoptosis 2008; 13 (1): 1-9.

12. Zaitone SA, Abo-Gresha NM. Rosuvastatin promotes angiogenesis and reverses isoproterenol-induced acute myocardial infarction in rats: role of iNOS and VEGF. Eur J Pharmacol 2012; 691 (1): 134-142.

13. Shamsaei N, Khaksari M, Erfani S, Rajabi H, Aboutaleb N. Exercise preconditioning exhibits neuroprotective effects on hippocampal CA1 neuronal damage after cerebral ischemia. Neural Regener Res 2015; 10 (8): 1245.

14. Schmittgen TD, Livak KJ. Analyzing real-time PCR data by the comparative CT method. Nature Protocols 2008; 3 (6): 1101-1108.

15. Mattson MP, Wan R. Beneficial effects of intermittent fasting and caloric restriction on the cardiovascular and cerebrovascular systems. J Nutrit Biochem 2005; 16 (3): 129-137.

16. Terman A, Brunk UT. Autophagy in cardiac myocyte homeostasis, aging, and pathology. Cardiovasc Res 2005; 68 (3): 355-365.

17. Han X, Turdi S, Hu N, Guo R, Zhang Y, Ren J. Influence of long-term caloric restriction on myocardial and cardiomyocyte contractile function and autophagy in mice. J Nutrit Biochem 2012; 23 (12): 1592-1599.

18. Wohlgemuth SE, Julian D, Akin DE, Fried J, Toscano K, Leeuwenburgh $\mathrm{C}$ et al. Autophagy in the heart and liver during normal aging and calorie restriction. Rejuvenation Res 2007; 10 (3): 281-292.

19. Xie Z, Klionsky DJ. Autophagosome formation: core machinery and adaptations. Nature Cell Biol 2007; 9 (10): 1102-1109.

20. Circu ML, Aw TY. Reactive oxygen species, cellular redox systems, and apoptosis. Free Rad Biol Med 2010; 48 (6): 749-762.

Received January 5, 2019. Accepted February 12, 2019. 